\title{
Erratum to: Effect of HSP90 inhibitor in pheochromocytoma PC12 cells: an experimental investigation
}

\author{
Yunze Xu • Chongyu Zhang • Dongning Chen • \\ Juping Zhao • Zhoujun Shen • Yuxuan Wu • Yu Zhu
}

Published online: 13 August 2013

(C) International Society of Oncology and BioMarkers (ISOBM) 2013

Erratum to: Tumor Biol.

DOI 10.1007/s13277-013-0996-4

The author's correction was misinterpreted by Springer. The correct author list is "Yunze Xu, Chongyu Zhang, Dongning Chen, Juping Zhao, Zhoujun Shen, Yuxuan Wu, Yu Zhu". Corrected author order can be seen above.

The online version of the original article can be found at http://dx.doi.org/ 10.1007/s13277-013-0996-4.

Y. Xu $\cdot$ C. Zhang $\cdot$ D. Chen $\cdot$ J. Zhao $\cdot$ Z. Shen $\cdot$ Y. Wu $\cdot$ Y. Zhu $(\bowtie)$

Department of Urology, Ruijin Hospital, School of Medicine,

Shanghai Jiaotong University, 197 Ruijin Er Road,

Shanghai 200025, China

e-mail: zyyyhyq@126.com 